\title{
HOMOGENEOUS ALMOST COMPLEX MANIFOLDS AND THEIR COMPACT QUOTIENTS
}

\author{
KANG-TAE KIM, KANG-HYURK LEE AND YOSHIKAZU NAGATA
}

\begin{abstract}
This paper investigates the (non)existence of compact quotients of the homogeneous almost-complex strongly-pseudoconvex manifolds discovered and classified by Gaussier-Sukhov [1, 2] and K.-H. Lee 3, 4].
\end{abstract}

\section{INTRODUCTION}

Let $M$ be an almost complex manifold of real dimension $2 m, m \geq 1$, with an almost complex structure $J$. It is said to be modeled after bounded strictly pseudoconvex domains, if the following two properties hold:

(1) $M$ is $J$-holomorphically equivalent to a subdomain of another almost complex manifold with its Levi form (cf. Section 2) at every boundary point positive-definite, and

(2) $M$ is Kobayashi hyperbolic.

It was generally believed for some time that, if one further assumes for it to be homogeneous, meaning that the action by the group $\operatorname{Aut}(M, J)$ of all $J$-holomorphic diffeomorphisms of $M$ into itself is transitive, then the almost complex structure should be integrable, and consequently - due to the well-known theorem by Wong and Rosay - the complex manifold $(M, J)$ would have to be biholomorphic to the unit ball $B^{m}$ in $\mathbb{C}^{m}$.

While such belief was justified in the case of $m \leq 2$ by Gaussier and Sukhov [1, 2], they in contrast showed that there exists an example indicating that it is not the case if $m>2$. Then the second named author of this article classified all such manifolds for every $m \geq 3$ in [3, 4] (cf. Theorem 2.1). It has turned out that there are infinitely many such examples, $J$-holomorphically inequivalent to each other.

Upon such observation, there arises a natural question whether the homogeneous manifolds (noncompact) obtained here would admit a compact quotient by a discrete subgroup. This question was asked to the first named author several different times by many prominent mathematicians, from at least 10 years ago, when he gave lectures explaining this line of research at the institutions including l'École Polytechnique de Palaiseau of France, Peking University of China, the Korea Institute for Advanced Study (Seoul) of Korea, and other places. It is our pleasure to acknowledge our indebtedness.

2010 Mathematics Subject Classification. 32M05.

Key words and phrases. J-automorphism, compact quotient.

Research of the authors is supported in part by the NRF Grant 2011-0030044 (SRC-GAIA) of The Republic of Korea. 
The purpose of this article is, indeed, to provide the answer-negative, however. Deferring the introduction of terminology and necessary definitions to the later sections, we present our results first.

Theorem 1.1. Let $\Omega$ be an open connected subset of an almost complex manifold $(M, J)$ of real dimension $2 m$ with $\mathcal{C}^{2}$ smooth strictly pseudoconvex boundary. If there exists a discrete subgroup $\Gamma$ of the automorphism group $\operatorname{Aut}(\Omega, J)$ such that $\Omega / \Gamma$ is compact, then $(\Omega, J)$ is biholomorphic to the standard unit ball in $\mathbb{C}^{m}$.

From here on, open connected subsets of a manifold will be called domains, as usual. Now, notice that Theorem 1.1 implies, according to the discussion above, the following result concerning the nonexistence of compact quotients.

Theorem 1.2. If $\Omega$ is a homogeneous domain with $\mathcal{C}^{2}$ smooth strictly pseudoconvex boundary in an almost complex manifold $(M, J)$ of real dimension $2 m$ whose $J$ structure is non integrable, then $(\Omega, J)$ does not admit any compact quotient by any discrete subgroup of $\operatorname{Aut}(\Omega, J)$.

We remark that these theorems are significant especially for the case $m \geq 3$.

\section{MODEL MANiFOLDS/DOMAINS}

For a domain $\Omega$ in $(M, J)$, any $\mathcal{C}^{\infty}$ smooth diffeomorphism $\psi: \Omega \rightarrow \Omega$ is called a J-holomorphic automorphism if $J \circ d \psi=d \psi \circ J$. (More generally, a smooth map $f:(M, J) \rightarrow(\tilde{M}, \tilde{J})$ between two almost complex manifolds is called pseudoholomorphic or, more precisely, $(J, \tilde{J})$-holomorphic if $\tilde{J} \circ d f=d f \circ J$ holds.) Such automorphisms form a topological group, denoted by $\operatorname{Aut}(\Omega, J)$ under the law of composition endowed with the compact-open topology.

Moreover, if the boundary $\partial \Omega$ of $\Omega$ is $\mathcal{C}^{2}$ smooth, then the implicit function theorem implies that for every boundary point $p$ there are an open neighborhood $V$ of $M$ and a $\mathcal{C}^{2}$ function $\rho: V \rightarrow \mathbb{R}$ such that $\Omega \cap V=\{z \in V: \rho(z)<0\}$ and that $d \rho(q) \neq 0$ for any $q \in \partial \Omega \cap V$. Such $\rho$ is called a local defining function.

For a 1 -form $\omega$, the dual $J^{*}$ of $J$ is defined by $\left(J^{*} \omega\right)(v)=\omega(J v)$. Then the Levi form of $\rho$ is defined to be

$$
\mathcal{L}_{\rho}(v, w):=-d\left(J^{*} d \rho\right)(v, J w) .
$$

Then we say that $\Omega$ is strictly $J$-pseudoconvex at $p \in \partial \Omega$ if $\mathcal{L}_{\rho}(v, v)>0$ for every nonzero vector $v \in T_{p} \partial \Omega \cap J T_{p} \partial \Omega$.

Now write $m=n+1 \geq 1$, let $z^{0}, z^{1}, \ldots, z^{n}$ represent the standard coordinate functions of $\mathbb{C}^{m}=\mathbb{C}^{n+1}$, and denote by $z^{\prime}=\left(z^{1}, \ldots, z^{n}\right)$ the standard coordinate system of $\mathbb{C}^{n}$.

For a mapping $\phi$ to $\mathbb{C}^{n+1}$, denote by $\phi^{0}=z^{0} \circ \phi$ and $\phi^{\prime}=z^{\prime} \circ \phi$, and hence $\phi=\left(\phi^{0}, \phi^{\prime}\right)$. Greek indices $\alpha, \beta, \ldots$ run from 1 to $n$ and the summation convention is always assumed: $\mathcal{N}_{\alpha \beta} z^{\beta}=\sum_{\beta=1}^{n} \mathcal{N}_{\alpha \beta} z^{\beta}$, for instance. We also put bar on the indices to denote the complex conjugation of the corresponding tensor coefficients such as: $\mathcal{N}_{\bar{\alpha} \bar{\beta}}=\overline{\mathcal{N}_{\alpha \beta}}, A_{\bar{\beta}}^{\bar{\alpha}}=\overline{A_{\beta}^{\alpha}}$.

Let $\mathbf{H}=\left\{\left(z^{0}, z^{\prime}\right) \in \mathbb{C} \times \mathbb{C}^{n}: \operatorname{Re} z^{0}+\left\|z^{\prime}\right\|^{2}<0\right\}$ be the Siegel half space. But we shall endow an almost complex structure potentially different from the standard integrable one. 
For each $n \times n$ skew-symmetric matrix $\mathcal{N}=\left(\mathcal{N}_{\alpha \beta}\right)$, define the almost complex structure $J_{\mathcal{N}}$ of $\mathbb{C}^{n+1}$ by

$$
\begin{aligned}
J_{\mathcal{N}}= & i \frac{\partial}{\partial z^{0}} \otimes d z^{0}+\left(i \frac{\partial}{\partial z^{\alpha}}+2 \mathcal{N}_{\alpha \beta} z^{\beta} \frac{\partial}{\partial z^{\overline{0}}}\right) \otimes d z^{\alpha} \\
& -i \frac{\partial}{\partial z^{\overline{0}}} \otimes d z^{\overline{0}}+\left(-i \frac{\partial}{\partial z^{\bar{\alpha}}}+2 \mathcal{N}_{\bar{\alpha} \bar{\beta}} z^{\bar{\beta}} \frac{\partial}{\partial z^{0}}\right) \otimes d z^{\bar{\alpha}} .
\end{aligned}
$$

Note that the almost complex structure $J_{\mathcal{N}}$ is integrable if and only if $\mathcal{N}=0$.

Then the following characterization of the strictly $J$-pseudoconvex domains with an automorphism orbit accumulating at a boundary point has been established earlier, by the second named author of this article:

Theorem 2.1 (4). If $(\Omega, J)$ is a domain in an almost complex manifold $(M, J)$ with $\mathcal{C}^{2}$ smooth strictly pseudoconvex boundary point $q \in \partial \Omega$ such that there are a point $p \in \Omega$ and a sequence of $J$-automorphisms $\varphi_{j} \in \operatorname{Aut}(\Omega, J)$ satisfying $\lim _{j \rightarrow \infty} \varphi_{j}(p)=q$, then $(\Omega, J)$ is $\left(J, J_{\mathcal{N}}\right)$-biholomorphic to one of $\left(\mathbf{H}, J_{\mathcal{N}}\right)$.

Remark 2.2. Every $\left(\mathbf{H}, J_{\mathcal{N}}\right)$ is homogeneous, as one sees in the next section.

\section{Automorphisms of $\left(\mathbf{H}, J_{\mathcal{N}}\right)$}

Note that, due to the preceding discussion, the proof of Theorem 1.1 reduces to demonstrating the (non)existence of the compact quotients for the domains $\left(\mathbf{H}, J_{\mathcal{N}}\right)$, called the model domains in [3, 4].

There are four types of automorphisms generating the automorphism group of the model domain $\left(\mathbf{H}, J_{\mathcal{N}}\right)$ for $\mathcal{N} \neq 0$ :

(1) $\mathbb{R}$-action: For each $s \in \mathbb{R}$,

$$
\mathcal{T}_{s}=\left(z^{0}+i s, z^{\prime}\right) \in \operatorname{Aut}\left(\mathbf{H}, J_{\mathcal{N}}\right) .
$$

(2) $\mathbb{C}^{n}$-action: For each $w^{\prime} \in \mathbb{C}^{n}$, let us define

$$
h_{w^{\prime}}\left(z^{\prime}\right)=-\left\|w^{\prime}\right\|^{2}-2\left\langle z^{\prime}, \bar{w}^{\prime}\right\rangle+i\left(\mathcal{N}_{\alpha \beta} z^{\alpha} w^{\beta}+\mathcal{N}_{\bar{\alpha} \bar{\beta}} z^{\bar{\alpha}} w^{\bar{\beta}}\right) .
$$

Here $\langle\cdot, \cdot\rangle$ is the standard hermitian product of $\mathbb{C}^{n}:\left\langle z^{\prime}, \bar{w}^{\prime}\right\rangle=\delta_{\alpha \bar{\beta}} z^{\alpha} w^{\bar{\beta}}$. Then $\left(\mathbb{C}^{n},+\right)$ can be embedded into $\operatorname{Aut}\left(\mathbf{H}, J_{\mathcal{N}}\right)$ by

$$
w^{\prime} \longmapsto \mathcal{H}_{w^{\prime}}=\left(z^{0}+h_{w^{\prime}}\left(z^{\prime}\right), z^{\prime}+w^{\prime}\right) \in \operatorname{Aut}\left(\mathbf{H}, J_{\mathcal{N}}\right),
$$

since $\mathcal{H}_{w^{\prime}} \circ \mathcal{H}_{v^{\prime}}=\mathcal{H}_{w^{\prime}+v^{\prime}}$ for any $w^{\prime}, v^{\prime} \in \mathbb{C}^{n}$.

We remark in passing that the subgroup generated by $\mathcal{H}_{w^{\prime}}$ and $\mathcal{T}_{s}$ is in fact isomorphic to the Heisenberg group.

(3) Dilation: For each $t>0$, we have

$$
\mathcal{D}_{t}=\left(t z^{0}, t^{1 / 2} z^{\prime}\right) \in \operatorname{Aut}\left(\mathbf{H}, J_{\mathcal{N}}\right) .
$$

(4) Isotropy: Let $\mathrm{U}_{\mathcal{N}}$ be the set of $n \times n$ complex matrices $A=\left(A_{\beta}^{\alpha}\right)$ with

$$
\delta_{\alpha \bar{\beta}}=A_{\alpha}^{\mu} \delta_{\mu \bar{\nu}} A_{\bar{\beta}}^{\bar{\nu}} \quad \text { and } \quad \mathcal{N}_{\alpha \beta}=A_{\alpha}^{\mu} \mathcal{N}_{\mu \nu} A_{\beta}^{\nu},
$$

i.e., $I=A^{t} \bar{A}$ and $\mathcal{N}=A^{t} \mathcal{N} A$. Then $\mathrm{U}_{\mathcal{N}}$ can be realized as a compact subgroup of the unitary group $\mathrm{U}(n)$ which can be embedded into $\operatorname{Aut}\left(\mathbf{H}, J_{\mathcal{N}}\right)$ via

$$
A \quad \longmapsto \quad \mathcal{R}_{A}=\left(z^{0}, A z^{\prime}\right) \in \operatorname{Aut}\left(\mathbf{H}, J_{\mathcal{N}}\right) .
$$


Then we have:

Theorem $3.1([4])$. For each $\phi \in \operatorname{Aut}\left(\mathbf{H}, J_{\mathcal{N}}\right)$ with $\mathcal{N} \neq 0$, there is a unique choice for $t>0, s \in \mathbb{R}, w^{\prime} \in \mathbb{C}^{n}$ and $A \in \mathrm{U}_{\mathcal{N}}$ such that

$$
\phi=\mathcal{T}_{s} \circ \mathcal{H}_{w^{\prime}} \circ \mathcal{D}_{t} \circ \mathcal{R}_{A}=\left(t z^{0}+h_{w^{\prime}}\left(z^{\prime}\right)+i s, t^{1 / 2} A z^{\prime}+w^{\prime}\right) .
$$

\section{Discrete Subgroups AND the Limit Sets}

In this section, we consider only the case $\mathcal{N} \neq 0$.

Definition 4.1. Let $H$ be a subgroup of the automorphism group $\operatorname{Aut}\left(\mathbf{H}, J_{\mathcal{N}}\right)$. By the limit set of $H$ we mean the set $\Lambda(H)$ of all accumulation points of the orbits by $H$. Here, the limit set may contain the points at infinity.

Suppose that $\Gamma$ is a discrete subgroup of $\operatorname{Aut}\left(\mathbf{H}, J_{\mathcal{N}}\right)$. The aim of this section is to analyze the limit set $\Lambda(\Gamma)$, which will eventually lead us to the proof of Theorem 1.1 .

Choose $\phi_{\nu} \in \Gamma$ and write

$$
\phi_{\nu}(z)=\left(t_{\nu} z^{0}+h_{w_{\nu}^{\prime}}\left(z^{\prime}\right)+i s_{\nu}, t_{\nu}^{1 / 2} A_{\nu} z^{\prime}+w_{\nu}^{\prime}\right)
$$

for some $t_{\nu}>0, s_{\nu} \in \mathbb{R}, w_{\nu}^{\prime} \in \mathbb{C}^{n}$ and $A_{\nu} \in \mathrm{U}_{\mathcal{N}}$.

For $p=\left(p^{0}, p^{\prime}\right) \in \mathbf{H}$, we assume that $\phi_{\nu}(p) \rightarrow\left(q^{0}, 0^{\prime}\right)$ as $\nu \rightarrow \infty$, where $q^{0} \in$ $\{i s: s \in \mathbb{R}\} \cup\{\infty\}$. Since $A_{\nu}$ is an element of the compact group $\mathrm{U}_{\mathcal{N}}$, we may assume that $A_{\nu} \rightarrow A_{\infty}$ in $\mathrm{U}_{\mathcal{N}}$. We may assume that $t_{\nu}$ converges in $[0,+\infty]$, and also $s_{\nu}$ in $[-\infty,+\infty]$, respectively. Note that $w_{\nu}^{\prime}$ can also be assumed to converge in the one-point compactification of $\mathbb{C}^{n}$.

Case (1): $\left\|w_{\nu}^{\prime}\right\| \rightarrow \infty$. Since $t_{\nu}^{1 / 2} A_{\nu} p^{\prime}+w_{\nu}^{\prime} \rightarrow 0^{\prime} \in \mathbb{C}^{n}$ and $A_{\nu} \rightarrow A_{\infty}$, we have $t_{\nu} \rightarrow \infty$ and $t_{\nu}^{-1 / 2} w_{\nu}^{\prime} \rightarrow-A_{\infty} p^{\prime}$. Let us consider

$$
\begin{aligned}
\operatorname{Re} \phi_{\nu}^{0}(p)=t_{\nu} \operatorname{Re} p^{0}-\| & w_{\nu}^{\prime} \|^{2}-2 \operatorname{Re}\left\langle p^{\prime}, \bar{w}_{\nu}^{\prime}\right\rangle \\
& =t_{\nu}\left(\operatorname{Re} p^{0}-\left\|t_{\nu}^{-1 / 2} w_{\nu}^{\prime}\right\|^{2}-2 \operatorname{Re}\left\langle t_{\nu}^{-1 / 2} p^{\prime}, t_{\nu}^{-1 / 2} \bar{w}_{\nu}^{\prime}\right\rangle\right) .
\end{aligned}
$$

Since $\operatorname{Re} p^{0}-\left\|t_{\nu}^{-1 / 2} w_{\nu}^{\prime}\right\|^{2}-2 \operatorname{Re}\left\langle t_{\nu}^{-1 / 2} p^{\prime}, t_{\nu}^{-1 / 2} \bar{w}_{\nu}^{\prime}\right\rangle \rightarrow \operatorname{Re} p^{0}-\left\|p^{\prime}\right\|^{2}<0$, we have $\phi_{\nu}^{0}(p) \rightarrow \infty$. Thus the accumulating point is $\left(\infty, 0^{\prime}\right)$.

Case (2): $w_{\nu}^{\prime} \rightarrow w_{\infty}^{\prime} \neq 0^{\prime}$. Since $t_{\nu}^{1 / 2} A_{\nu} p^{\prime}+w_{\nu}^{\prime} \rightarrow 0^{\prime} \in \mathbb{C}^{n}$ and $A_{\nu} \rightarrow A_{\infty}$, we have $t_{\nu} \rightarrow t>0$. If $s_{\nu} \rightarrow s \in \mathbb{R}$, then

$$
\begin{aligned}
\phi_{\nu}(z)= & \left(t_{\nu} z^{0}+h_{w_{\nu}^{\prime}}\left(z^{\prime}\right)+i s_{\nu}, t_{\nu}^{1 / 2} A_{\nu} z^{\prime}+w_{\nu}^{\prime}\right) \\
& \longrightarrow \quad \phi(z)=\left(t z^{0}+h_{w_{\infty}^{\prime}}\left(z^{\prime}\right)+i s, t^{1 / 2} A_{\infty} z^{\prime}+w_{\infty}^{\prime}\right) \in \operatorname{Aut}\left(\mathbf{H}, J_{\mathcal{N}}\right) .
\end{aligned}
$$

This is a contradiction to the discreteness of $\Gamma$. Hence $\left|s_{\nu}\right| \rightarrow \infty$ so that the accumulation point is $\left(\infty, 0^{\prime}\right)$ only.

Case (3): $w_{\nu}^{\prime} \rightarrow 0^{\prime}$. Suppose that $t_{\nu}$ does not converge to 0 . If $p^{\prime} \neq 0^{\prime}$, then $t_{\nu} \rightarrow 0$ because $t_{\nu}^{1 / 2} A_{\nu} p^{\prime}+w_{\nu}^{\prime} \rightarrow 0^{\prime} \in \mathbb{C}^{n}$. Thus we must have $p^{\prime}=0^{\prime}$ and $\operatorname{Re} p^{0}<0$. 
If $t_{\nu} \rightarrow \infty$, then

$$
\operatorname{Re} \phi_{\nu}^{0}(p)=t_{\nu} \operatorname{Re} p^{0}-\left\|w_{\nu}^{\prime}\right\|^{2}-2 \operatorname{Re}\left\langle p^{\prime}, \bar{w}_{\nu}^{\prime}\right\rangle=t_{\nu} \operatorname{Re} p^{0}-\left\|w_{\nu}^{\prime}\right\|^{2}
$$

and this implies that $\operatorname{Re} \phi_{\nu}^{0}(p) \rightarrow-\infty$. So the accumulation point is $\left(\infty, 0^{\prime}\right)$.

If $t_{\nu} \rightarrow t>0$, by the same argument as in Case (2) $\left|s_{\nu}\right| \rightarrow \infty$. Thus the accumulation point is $\left(\infty, 0^{\prime}\right)$.

It remains to analyze the case of $t_{\nu} \rightarrow 0$. For this purpose, we pose the following

Lemma 4.2. Let $\Gamma$ be a discrete subgroup of $\operatorname{Aut}\left(\mathbf{H}, J_{\mathcal{N}}\right)$. If there is $\phi \in \Gamma$ of the form $\phi(z)=\left(t z^{0}+i s, t^{1 / 2} A z^{\prime}\right)$ for some $t<1$, then each $\psi \in \Gamma$ is of the form $\psi(z)=\left(a z^{0}+i b, a^{1 / 2} B z^{\prime}\right)$ with $\frac{b}{1-a}=\frac{s}{1-t}$.

Postponing the proof of this lemma to the end of this section, we continue analyzing the accumulation points.

Assume that $t_{\nu} \rightarrow 0$ as $\nu \rightarrow \infty$. Then fix $\phi_{m}$ such that $t_{m}<1$ and, consequently, $I-\sqrt{t_{m}} A_{m}$ is invertible. From now on, we simply denote by $t=t_{m}, s=s_{m}$, $w^{\prime}=w_{m}^{\prime}, A=A_{m}$ and put $\zeta^{\prime}=(I-\sqrt{t} A)^{-1} w^{\prime}$ and $\gamma=\mathcal{H}_{\zeta^{\prime}} \circ \mathcal{D}_{t} \circ \mathcal{R}_{A}$. Thus we have

$$
\begin{aligned}
\gamma^{\prime}\left(z^{\prime}\right) & =t^{1 / 2} A z^{\prime}+\zeta^{\prime} \\
\left(\gamma^{-1}\right)^{\prime}\left(z^{\prime}\right) & =t^{-1 / 2} A^{-1}\left(z^{\prime}-\zeta^{\prime}\right),
\end{aligned}
$$

which immediately implies

$$
\gamma^{-1} \circ \phi_{m} \circ \gamma(z)=\left(t z^{0}+i \tilde{s}, t^{1 / 2} A z^{\prime}\right),
$$

for some $\tilde{s} \in \mathbb{R}$. For general integer values of $\nu$, it follows that

$$
\begin{aligned}
& \left(\gamma^{-1} \circ \phi_{\nu} \circ \gamma\right)^{\prime}\left(z^{\prime}\right)=\left(\gamma^{-1}\right)^{\prime} \circ \phi_{\nu}^{\prime} \circ \gamma^{\prime}\left(z^{\prime}\right) \\
& \quad=t_{\nu}^{1 / 2} A^{-1} A_{\nu} A z^{\prime}+t^{-1 / 2} t_{\nu}^{1 / 2} A^{-1} A_{\nu} \zeta^{\prime}+t^{-1 / 2} A^{-1} w_{\nu}^{\prime}-t^{-1 / 2} A^{-1} \zeta^{\prime} .
\end{aligned}
$$

Since $\gamma^{-1} \circ \phi_{m} \circ \gamma$ is of the same form as $\phi$ in Lemma 4.2, we obtain, as far as the element $\gamma^{-1} \circ \phi_{\nu} \circ \gamma$ of the discrete group $\gamma^{-1} \Gamma \gamma$ is concerned, that

$$
\left(\gamma^{-1} \circ \phi_{\nu} \circ \gamma\right)^{\prime}\left(z^{\prime}\right)=t_{\nu}^{1 / 2} C_{\nu} z^{\prime}
$$

where $C_{\nu}=A^{-1} A_{\nu} A$. Now we have

$$
\begin{aligned}
\left(\phi_{\nu}\right)^{\prime}\left(z^{\prime}\right) & =\gamma^{\prime} \circ\left(\gamma^{-1} \circ \phi_{\nu} \circ \gamma\right)^{\prime} \circ\left(\gamma^{-1}\right)^{\prime}(z) \\
& =t_{\nu}^{1 / 2} A C_{\nu} A^{-1} z^{\prime}-t_{\nu}^{1 / 2} A C_{\nu} A^{-1} \zeta^{\prime}+\zeta^{\prime} \longrightarrow \zeta^{\prime}
\end{aligned}
$$

as $\nu \rightarrow \infty$ for any $z^{\prime} \in \mathbb{C}^{n}$. Since $\left(\phi_{\nu}\right)^{\prime}\left(p^{\prime}\right) \rightarrow 0^{\prime}$ as $\nu \rightarrow \infty$, it follows that $\zeta^{\prime}=0$, which in turn implies $w^{\prime}=w_{m}^{\prime}=0$. Thus $\phi_{m}(z)=\left(t z^{0}+i s, t^{1 / 2} A z^{\prime}\right)$ with $t<1$. Applying Lemma 4.2 again for $\phi=\phi_{m}$, each $\psi \in \Gamma$ is of the form

$$
\psi(z)=\left(a z^{0}+i b, a^{1 / 2} B z^{\prime}\right)
$$

with

Especially,

$$
\frac{b}{1-a}=\frac{s}{1-t} .
$$

$$
\phi_{\nu}(z)=\left(t_{\nu} z^{0}+i s_{\nu}, t_{\nu}^{1 / 2} A_{\nu} z^{\prime}\right)
$$


where

$$
\frac{s_{\nu}}{1-t_{\nu}}=\frac{s}{1-t}
$$

for any $\nu$. Thus

$$
s_{\nu}=\frac{s\left(1-t_{\nu}\right)}{1-t} \longrightarrow \frac{s}{1-t}
$$

SO

$$
\phi_{\nu}(z)=\left(t_{\nu} z^{0}+i s_{\nu}, t_{\nu}^{1 / 2} A_{\nu} z^{\prime}\right) \rightarrow\left(i s /(1-t), 0^{\prime}\right) .
$$

Since $s /(1-t)$ is an invariant of $\Gamma$, the accumulation point should be $\left(i s /(1-t), 0^{\prime}\right)$ when $w_{\nu}^{\prime} \rightarrow 0^{\prime}$ and $t_{\nu} \rightarrow 0$.

Proof of Lemma 4.2. Since $\phi^{-1}(z)=\left(t^{-1} z^{0}-t^{-1} i s, t^{-1 / 2} A^{-1} z^{\prime}\right)$, we have

$$
\begin{aligned}
\phi^{k}(z) & =\left(t^{k} z^{0}+i s \sigma_{k}, t^{k / 2} A^{k} z^{\prime}\right) \\
\phi^{-k}(z) & =\left(t^{-k} z^{0}-i s t^{-k} \sigma_{k}, t^{-k / 2} A^{-k} z^{\prime}\right)
\end{aligned}
$$

where $\phi^{k}$ is the $k$-th iteration of $\phi$ and $\phi^{-k}=\left(\phi^{k}\right)^{-1}$ and

$$
\sigma_{k}=1+t+\cdots+t^{k-1}
$$

Take $\psi \in \Gamma$ and let

$$
\psi(z)=\left(a z^{0}+h_{v^{\prime}}\left(z^{\prime}\right)+i b, a^{1 / 2} B z^{\prime}+v^{\prime}\right)
$$

Then

$$
\begin{aligned}
& \phi^{k} \circ \psi \circ \phi^{-k}(z) \\
= & \left(a z^{0}+i\left((1-a) s \sigma_{k}+t^{k} b\right)+t^{k} h_{v^{\prime}}\left(t^{-k / 2} A^{-k} z^{\prime}\right), a^{1 / 2} A^{k} B A^{-k} z^{\prime}+t^{k / 2} A^{k} v^{\prime}\right) .
\end{aligned}
$$

Since $t^{k} h_{v^{\prime}}\left(t^{-k / 2} A^{-k} z^{\prime}\right) \rightarrow 0$ as $k \rightarrow \infty, \phi^{k} \circ \psi \circ \phi^{-k}$ subsequentially converges to some $\tilde{\psi} \in \Gamma$ of the form

$$
\tilde{\psi}=\left(a z^{0}+(1-a) i s \sigma, a^{1 / 2} \widetilde{A} B \widetilde{A}^{-1} z^{\prime}\right)
$$

where $\sigma=\lim \sigma_{k}=1 /(1-t)$. Since $\Gamma$ is discrete, we conclude that $v^{\prime}=0$ and

$$
(1-a) s \sigma_{k}+t^{k} b=(1-a) s \sigma
$$

for infinitely many $k$. Thus

$$
\frac{b}{1-a}=\frac{1}{t^{k}} s\left(\sigma-\sigma_{k}\right)=s \sigma=\frac{s}{1-t} .
$$

This completes the proof. 


\section{Proof of Theorems 1.1 and 1.2}

Let $\Gamma$ be a discrete subgroup of $\operatorname{Aut}(\Omega, J)$ with $\Omega / \Gamma$ compact. Then there exists a (finite) boundary point at which the orbit of $\Gamma$ accumulates. By Theorem 2.1, $\Omega$ is biholomorphic to a model $\left(\mathbf{H}, J_{\mathcal{N}}\right)$, say, that is homogeneous and complete Kobayashi hyperbolic. Suppose now that $\Omega$, or equivalently $\left(\mathbf{H}, J_{\mathcal{N}}\right)$, is not biholomorphic to the standard unit ball of $\mathbb{C}^{m}$.

According to the arguments of Section 4 above, the set $\Lambda(\Gamma) \cap(\mathbb{C} \times\{(0, \ldots, 0)\})$ consists of at most two elements: the extended boundary point $(\infty, 0, \ldots, 0)$, or the boundary point of type $(a, 0, \ldots, 0)$ for some $a \in \mathbb{C}$. Suppose that $\left(\mathbf{H}, J_{\mathcal{N}}\right)$ admits a compact quotient by a discrete group $\Gamma$, say. Then, since $\left(\mathbf{H}, J_{\mathcal{N}}\right)$ is complete Kobayashi hyperbolic, we must have

$$
\Lambda(\Gamma) \cap(\mathbb{C} \times\{(0, \ldots, 0)\})=\partial \mathbf{H} \cap(\mathbb{C} \times\{(0, \ldots, 0)\})=\mathbb{R} \times\{(0, \ldots, 0)\},
$$

which cannot coincide with any two element set. This yields the proof of Theorems 1.1 as well as 1.2 .

\section{REFERENCES}

[1] H. Gaussier; A. Sukhov: WongRosay theorem in almost complex manifolds, http: www.arXiv.org: math.CV/0307335

[2] H. Gaussier; A. Sukhov: On the geometry of model almost complex manifolds with boundary, Math. Z. 254 (2006), no. 3, 567-589.

[3] K.-H. Lee: Domains in almost complex manifolds with an automorphism orbit accumulating at a strongly pseudoconvex boundary point. Michigan Math. J. 54 (2006), no. 1, 179-205.

[4] K.-H. Lee: Strongly pseudoconvex homogeneous domains in almost complex manifolds. $J$. Reine Angew. Math. 623 (2008), 123-160.

[5] J.-P. Rosay: Sur une caractrisation de la boule parmi les domaines de $\mathbf{C}^{n}$ par son groupe d'automorphismes. (French) Ann. Inst. Fourier (Grenoble) 29 (1979), no. 4, ix, 91-97.

[6] B. Wong: Characterization of the unit ball in $\mathbb{C}^{n}$ by its automorphism group. Invent. Math. 41 (1977), no. 3, 253-257.

Kang-Tae Kim and Yoshikazu Nagata: Department of Mathematics and Center for Geometry and its Applications, Pohang University of Science and Technology, 37673, The Republic of Korea

E-mail address: kimkt@postech.ac.kr, yoshikazu@postech.ac.kr

Kang-Hyurk Lee: Department of Mathematics and Research Institute of Natural Science, Gyeongsang National University, Jinju, Gyeongnam, 660-701, The Republic of Korea

E-mail address: nyawoo@gnu.ac.kr 